\title{
Rehabilitation for children and adolescents after cancer: importance and implementation in Austria
}

\author{
Gustav Fischmeister - David Riedl - Gabriele Sanio - Thomas Bogendorfer - Bernhard Holzner • \\ Gerhard Rumpold · Alain Nickels · Thomas Licht · Wolfgang Sperl
}

Received: 12 May 2021 / Accepted: 17 June 2021 / Published online: 23 July 2021

(C) The Author(s) 2021

\begin{abstract}
Summary Until 2018 only adults had access to rehabilitation in Austria, but since then 5 centers for pediatric rehabilitation with different indications have been established with the goal of improving the health of sick children and young adults. The pediatric rehabilitation center "Leuwaldhof", which is located south of Salzburg, is the only pediatric oncologic rehabilitation center in Austria. It offers rehabilitation and recovery for pediatric patients who suffered from malignancies, as well as for their families and siblings, but also for acute or chronic disease in metabolism or digestion. Cancer and its treatment significantly decrease the quality of life (QoL) of pediatric patients and their families. Families often have to split up during the months of chemotherapy if there are siblings in the family and very often it is the mother who stays with the sick child in the hospital. To facilitate recovery for the families in these difficult times, interdisciplinary and family-oriented inpatient rehabilitation
\end{abstract}

\footnotetext{
G. Fischmeister $(\bowtie) \cdot$ G. Sanio · T. Bogendorfer · A. Nickels . T. Licht

Rehabilitation St. Veit, Leuwaldhof, St. Veiter

Straße 48, 5621 St. Veit im Pongau, Austria

gustav.fischmeister@reha-stveit.at
}

D. Riedl · G. Rumpold

Department of Medical Psychology, Medical University of Innsbruck, Anichstraße 35, 6020 Innsbruck, Austria

\section{B. Holzner}

Universitätsklinik für Psychiatrie II, Medical University of Innsbruck, Anichstraße 35, 6020 Innsbruck, Austria

\section{W. Sperl}

Paracelsus Medical University,

Strubergasse 21, 5020 Salzburg, Austria

B. Holzner · G. Rumpold

Evaluation Software Development (ESD), Valiergasse 60-62, 6020 Innsbruck, Austria has recently been implemented in Austria. To evaluate the improvements during the rehabilitation, the QoL of the patients and families has been routinely assessed since the opening of the center. In a specifically designed 'life app', patients and families complete the Pediatric Quality of Life Inventory (PedsQL; generic score and cancer module) before and after rehabilitation on their own electronic devices. Data of 98 patients and 124 parents between June 2018 and December 2019 show significant improvements in QoL. Our goal is to support the children and their families to help them return to normal life. Our results show rehabilitation helps achieve this important goal.

Keywords Quality of life · Pediatric · Hematooncology $\cdot$ Leuwaldhof · Austria

Abbreviations

CHES Computer-based Health Evaluation System

ESD Evaluation Software Development

FOR Family-oriented Rehabilitation

PedsQL Pediatric QoL

PRO Patient-reported Outcome

QoL Quality of Life

\section{Importance}

In Austria, approximately 300 new pediatric patients are diagnosed each year with various types of cancer, at least $80 \%$ survive and many of them would benefit from rehabilitation. They have suffered psychological trauma (life-threatening diagnosis), operations, loss of function and will have to cope with disability and late effects during the rest of their lives [1]. Both the patients and their families are often emotionally and physically exhausted due to the disease and its treatment, which is frequently associated with severe vom- 
iting, nausea, pain, infection and fear of losing a loved one [2].

According to the World Health Organization, "rehabilitation is a process aimed at enabling patients to reach and maintain their optimal physical, sensory, intellectual, psychological and social functional level" [3].

According to the United Nations Convention on the Rights of the Child, article 6, children have the right "to survive and develop healthily" [4]. The following is stated in article 23: "children who have any kind of disability have the right to special care and support ... so that they can live full and independent lives" [4]. Thus, although the term "rehabilitation" is used only in article 39 (Rehabilitation of Child Victims), the United Nations Convention clearly expresses that children should have access to rehabilitative measures in the event of relevant underlying health problems [3-5].

In Germany, activities to establish pediatric familyoriented rehabilitation started in 1980 and since 1985 four pediatric family-oriented rehabilitation centers (Bad Oexen Brinkmeier, Katharinenhöhe, Tannheim and Syltklinik) offer pediatric rehabilitation for affected German families [6]. Due to the lack of a center for pediatric cancer rehabilitation in Austria, children were referred from Austria to these German rehabilitation centers until 2018.

The goal of German rehabilitation centers is for families to meet and exchange experiences with each other, and for adolescents to meet and learn from peer groups, while being guided by experienced experts and protected in a relaxing surrounding during a 4week-long stay with daily 24-hour service. The costs are covered by the German Retirement Fund and the patient's National Health Insurance. Special training is provided on transferring health information into selfmanagement and health competence. The success of rehabilitative measure includes medical, psychological, pedagogic, social, and nutritional training to improve various aspects of patients' lives [7].

To help the families recover in these difficult times, rehabilitation has also been implemented in Austria. Children need a healthy and caring surrounding, especially after a malignant disease, to recover and stay healthy. Our goal is to strengthen the children and their families, to achieve resilience and to return to a normal life after life-threatening disease, while having learned to be strong as a lion, proud to have fought and survived, stronger than ever before. The aim of this study was to present key elements of family-oriented rehabilitation in Austria as well as first results from our routine QoL assessment before and after rehabilitation.

\section{Implementation}

In Austria the first positioning paper was written in 2001, which ultimately led to the first step toward "pediatric rehabilitation" in the Austrian "Gesundheitsplan” in 2004. In 2010, a working group for pediatric rehabilitation was established in the Austrian pediatric society. A team of distinguished pediatricians was assigned with the calculation of bed capacities, which were estimated to be between 340 and 450 beds. Together with many other specialists, a father whose daughter had leukemia, and with the help of many other organizations and specialized hospitals, a decision for public financing of pediatric rehabilitation in Austria was made in the year of 2015. Owing to the small patient numbers only one of the 6 potential centers was assigned for pediatric hematooncologic patients, in order to better focus the competence and quality [8].

In June 2018 the first pediatric rehabilitation center in Austria for patients and their families with hematooncologic malignancies-"Leuwaldhof" (the lion forest court)—was opened. Leuwaldhof has 20 beds for pediatric patients and 50 beds for the families of the primary patients having suffered malignancies. Furthermore, there are 12 beds for patients with diseases associated with metabolism and digestion. It is connected to an adult oncology rehabilitation institution, which creates the possibility for young adults with malignancies to bring their children and meet youth and other young families from the pediatric rehabilitation center.

Our rehabilitation center provides psychological support, dietary counseling (with a focus on healthy eating behavior and weight gain), physiotherapy (with a focus on improving physical functioning and correction of motor dysfunction), and social work to prevent unemployment.

Kindergarten and schoolteachers help the patients and their siblings to catch up with their school class and friends. Because young cancer survivors need a healthy and caring surrounding, the whole family receives therapy (parents and siblings $90 \mathrm{~min}$, the primary patient $150 \mathrm{~min}$ ) from Monday to Friday for 4 weeks. On Saturday's, trips are arranged by recreational educators, who are also available during the week after therapy until the evening.

\section{Materials and methods}

For collecting age- and diagnosis-related patient reported outcomes (PRO), we used an eHealth App, called "Life App", which based on patient's responses documents changes of wellbeing during 4 weeks of pediatric rehabilitation, and a 12-month follow-up. The "Life App" was designed by ESD (Evaluation Software Development GmbH in Innsbruck, Austria) after years of experience in research, evaluation, teaching and administration both in university and education institutions. The method was described by Holzner et al. in 2012 [9]. 


\section{Procedure}

Baseline assessment (T0) was performed prior to or at admission to the rehabilitation center, at the end of the stay (T1). Children older than 5 years completed the self-report version of the questionnaires and parents additionally and independently completed a proxy-version of the questionnaires.

\section{Measures}

The assessment of health-related quality of life (HRQOL) was conducted using the Pediatric Quality of Life Inventory (PedsQL) 4.0 Generic Core Scales and the PedsQL 3.0 Cancer Module. The PedsQL 4.0 Generic Core Scales consists of 23 items, which assess six functioning subscales (physical, emotional, social, school, psychosocial), and a total score [10]. The PedsQL 3.0 Cancer Module consists of 27 items to assess eight symptom subscales (pain and hurt; nausea; procedural anxiety; treatment anxiety; worry; cognitive problems; perceived physical appearance; communication) and a total score [11]. Both questionnaires are available in age-specific versions and offer a patient self-report and an observer/proxy version. Higher values on both measures represent better outcome (i.e., higher functioning and lower symptom load) [10, $11]$.

\section{Statistical analysis}

Data collected between June 2018 and December 2020 were included in the analysis. Changes of HRQOL as a result of the rehabilitation were analyzed using repeated measures analysis of variance (ANOVA). Analyses were conducted for the patient self-reports and parent proxy reports separately. Partial eta squared $\left(\eta^{2}\right)$ was calculated to estimate the effect size of the mean differences concerning the PedsQL total scores and subscales. Values of $\eta^{2}=0.01, \eta^{2}=0.06$, and $\eta^{2}=0.14$ were considered as small, medium, and large, respectively [12]. $P$ values $<0.05$ were considered statistically significant. All calculations were conducted with IBM SPSS - Statistics for Windows (v21; IBM Corp., Armonk, NY, USA).

\section{Results}

A total of 98 children with cancer and 124 corresponding parents completed the assessment before and after rehabilitation. Mean age of the children was 11.8 $( \pm 4.0$; range $5-18$ ) years and $58.1 \%$ were male.

Children reported statistically significant improvements in terms of their physical $(p<0.001)$, emotional $(p=0.005)$, school $(p<0.001)$, and psychosocial functioning $(p=0.001)$. However, no significant improvement for cancer-related symptoms was reported by the children (all $p>0.05$ ). The parents had a tendency to report worse scores for both functioning and symptom scores at baseline, but also reported a notably more pronounced improvement of the

Table 1 Self-reported quality of life before (TO) and after (T1) rehabilitation (child report)

\begin{tabular}{|c|c|c|c|c|c|}
\hline & \multicolumn{5}{|l|}{$\begin{array}{l}\text { Child sample } \\
(n=98)\end{array}$} \\
\hline & T0 & \multirow{2}{*}{$\begin{array}{l}\text { T1 } \\
\text { Mean (SD) }\end{array}$} & \multicolumn{3}{|l|}{ Mean diff } \\
\hline & Mean (SD) & & (T1-T0) & $p$-value & $\eta^{2}$ \\
\hline \multicolumn{6}{|l|}{ PedsQL Generic QoL } \\
\hline Physical functioning & 73.7 (18.4) & $81.1(15.6)$ & 7.4 & $<0.001$ & 0.167 \\
\hline Emotional functioning & $75.3(19.8)$ & $80.7(18.7)$ & 5.4 & 0.005 & 0.079 \\
\hline Social functioning & $78.6(18.1)$ & $81.9(18.6)$ & 3.3 & 0.086 & 0.030 \\
\hline School functioning & $67.9(17.9)$ & $75.1(19.0)$ & 7.2 & $<0.001$ & 0.122 \\
\hline Psychosocial functioning & $73.9(14.6)$ & $79.3(15.6)$ & 5.4 & 0.001 & 0.104 \\
\hline Total score & $73.8(13.9)$ & $79.9(14.3)$ & 6.1 & $<0.001$ & 0.145 \\
\hline \multicolumn{6}{|l|}{ PedsQL Cancer Module } \\
\hline Pain and hurt & 75.4 (23.2) & $79.5(22.5)$ & 4.1 & 0.118 & 0.025 \\
\hline Nausea & $77.4(19.0)$ & $80.4(17.5)$ & 3 & 0.123 & 0.024 \\
\hline Procedural anxiety & $74.3(30.7)$ & $77.9(30.0)$ & 3.6 & 0.168 & 0.019 \\
\hline Treatment anxiety & $86.2(21.4)$ & $87.9(20.6)$ & 1.7 & 0.467 & 0.005 \\
\hline Worry & $75.8(24.3)$ & $78.2(21.9)$ & 2.4 & 0.214 & 0.016 \\
\hline Cognitive problems & 74.4 (21.6) & $77.5(21.3)$ & 3.1 & 0.132 & 0.023 \\
\hline Perceived physical appearance & $81.2(20.8)$ & $80.3(20.6)$ & -0.9 & 0.652 & 0.002 \\
\hline Communication & $78.8(23.9)$ & $82.7(24.1)$ & 3.9 & 0.101 & 0.027 \\
\hline Total score & 77.8 (13.3) & $80.4(15.4)$ & 2.6 & 0.087 & 0.030 \\
\hline
\end{tabular}


Table 2 Proxy-reported quality of life before (T0) and after (T1) rehabilitation (parents report)

\begin{tabular}{|c|c|c|c|c|c|}
\hline & \multicolumn{5}{|l|}{$\begin{array}{l}\text { Parent sample } \\
(n=124)\end{array}$} \\
\hline & \multirow{2}{*}{$\begin{array}{l}\text { T0 } \\
\text { Mean (SD) }\end{array}$} & $\mathrm{T} 1$ & \multicolumn{3}{|l|}{ Mean diff } \\
\hline & & Mean (SD) & (T1-T0) & $p$-value & ES \\
\hline \multicolumn{6}{|l|}{ PedsQL Generic QoL } \\
\hline Physical functioning & $68.6(20.9)$ & $75.8(21.0)$ & 7.2 & $<0.001$ & 0.168 \\
\hline Emotional functioning & $62.7(20.6)$ & $74.0(20.5)$ & 11.3 & $<0.001$ & 0.317 \\
\hline Social functioning & $73.2(18.2)$ & $78.0(19.8)$ & 4.8 & 0.001 & 0.088 \\
\hline School functioning & $68.0(22.6)$ & $74.6(23.4)$ & 6.6 & 0.001 & 0.086 \\
\hline Psychosocial functioning & $67.9(16.5)$ & $75.8(18.9)$ & 7.9 & $<0.001$ & 0.245 \\
\hline Total score & $68.2(16.7)$ & $75.8(18.6)$ & 7.6 & $<0.001$ & 0.257 \\
\hline \multicolumn{6}{|l|}{ PedsQL Cancer Module } \\
\hline Pain and hurt & 70.9 (26.2) & $78.9(23.4)$ & 8.0 & $<0.001$ & 0.125 \\
\hline Nausea & $72.8(20.6)$ & $77.9(20.8)$ & 5.1 & 0.001 & 105 \\
\hline Procedural anxiety & $53.4(36.3)$ & $68.1(34.9)$ & 14.7 & $<0.001$ & 0.225 \\
\hline Treatment anxiety & $78.6(23.5)$ & $83.5(22.9)$ & 4.9 & 0.007 & 0.057 \\
\hline Worry & $73.1(27.8)$ & $78.8(25.6)$ & 5.7 & 0.002 & 0.077 \\
\hline Cognitive problems & $67.4(21.6)$ & $69.5(24.0)$ & 2.1 & 0.33 & 0.007 \\
\hline Perceived physical appearance & $76.5(25.5)$ & $79.8(23.9)$ & 3.3 & 0.09 & 0.023 \\
\hline Communication & $56.5(29.7)$ & $55.0(31.3)$ & -1.5 & 0.54 & 0.003 \\
\hline Total score & $68.2(16.7)$ & $75.8(18.6)$ & 7.6 & $<0.001$ & 0.257 \\
\hline
\end{tabular}

children's physical $(p<0.001)$, emotional $(p<0.001)$, social $(p=0.001)$, psychosocial $(p<0.001)$, and school functioning $(p=0.001)$, with medium to large effect sizes. In addition, parents also observed significant improvements in terms of pain $(p<0.001)$, nausea $(p=0.001)$, procedural $(p<0.001)$ and treatment anxiety $(p=0.007)$ and general worries $(p=0.002)$. Details are provided in Tables 1 and 2.

\section{Discussion}

Survivors of childhood cancer and their families often are emotionally and physically exhausted after many months of therapy, which often includes severe symptoms such as nausea, pain, infections, and fear to losing a close person. Disturbances in relationships and family structure with psychosomatic symptoms may occur. Pediatric rehabilitation may facilitate the improvement of the patients and family's quality of life and may help to decrease symptoms in the treatment's aftermath [13]. However, data on effectiveness of pediatric oncological rehabilitation are still rare. Recent publications from Germany show evidence of the benefits of FOR. Not only the patient, but the siblings and the parents benefit; the core family must be included. Those who went through the cancer diagnosis and treatment together need to participate in the "healing process" of the family system in order to guarantee the rehabilitation success of the oncological patient [14].

The aim of this paper was to present data on treatment success during an inpatient rehabilitation stay at the pediatric oncologic rehabilitation center in Austria, the Leuwaldhof. Both the children and their parents reported significant improvements of the child's health-related QoL. The children reported the largest effects for physical functioning, school functioning and psychosocial functioning, while the parents observed the most pronounced improvements for emotional and psychosocial functioning as well as the child's procedural anxiety, i.e., the excessive fear of medical procedures which lead to acute distress and may interfere with necessary medical procedures. The positive outcomes of our study are in line with previous research, which has reported good functional improvement in pediatric oncology rehabilitation [15].

It is noteworthy that children did not report a significant improvement in regard to their disease- and treatment-related symptoms, while parents reported medium to large differences in more than half of the assessed symptoms. These results are in line with previous studies, which found notable differences in selfand proxy-report for children and parents, especially in regard to less observable items, such as pain or fatigue [16, 17]. Thus, parent proxy-report is recommended to complement, but not to substitute children's self-report.

Furthermore, the cancer module of the PedsQL has been designed for the acute phase of the anticancer treatment and maybe therefore less suitable for the rehabilitation setting because the qualities nausea, anxiety, worry etc. are mainly chemotherapy side effects and may not persist for very long after completion of 
chemotherapy. This highlights the need for specific survivor-focused questionnaires, inquiring less about acute symptoms and more about functioning after the acute phase of treatment.

Overall, our results are encouraging since they show significant improvements in almost all major aspects of the children's health-related QoL in the course of the rehabilitation treatment. Rehabilitation-adapted questionnaires should be developed focusing more on the habitational qualities of life, showing the achieved improvement in participation and independence during the stay and after one year.

\section{Take home message}

Family-oriented pediatric rehabilitation in hematooncology shows improvement in most qualities of life. It should be a regular part of the therapeutic regime for all patients after chemotherapy, transplantation and treatment for immune deficiency to create a healthy and caring surrounding for the children during their recovery.

Acknowledgements Rehabilitation St. Veit carried the costs of the statistical data analysis, which was performed by Gerhard Rumpold and David Riedl, with the software tool CHES, described under https://CHES.pro/. No other support/funding was reported.

\section{Declarations}

Conflict of interest G. Fischmeister, chief medical officer of the pediatric rehabilitation Leuwaldhof, is part of Rehabilitation St. Veit im Pongau, and T. Licht, G. Sanio, T. Bogendorfer and A. Nickels hold leading positions at rehabilitation St. Veit. W. Sperl is scientific medical advisor concerning metabolic diseases for the Leuwaldhof. B. Holzner and G. Rumpold hold intellectual property rights on the software CHES. D. Riedl declares that he has no competing interests.

Ethical standards The Ethics Commission of the State of Salzburg, Austria, has evaluated the study (administrative notice no. 415-EP/73/415-2014). Patients were included in the study upon written informed consent.

Open Access This article is licensed under a Creative Commons Attribution 4.0 International License, which permits use, sharing, adaptation, distribution and reproduction in any medium or format, as long as you give appropriate credit to the original author(s) and the source, provide a link to the Creative Commons licence, and indicate if changes were made. The images or other third party material in this article are included in the article's Creative Commons licence, unless indicated otherwise in a credit line to the material. If material is not included in the article's Creative Commons licence and your intended use is not permitted by statutory regulation or exceeds the permitted use, you will need to obtain permission directly from the copyright holder. To view a copy of this licence, visit http://creativecommons.org/licenses/by/4.0/.

\section{References}

1. Oeffinger KC, Mertens AC, Sklar CA, Kawashima T, Hudson MM, Meadows AT, et al. Chronic health condi- tions in adult survivors of childhood cancer. N Engl J Med. 2006;355(15):1572-82. https://doi.org/10.1056/ NEJMsa060185.

2. Kestler SA, LoBiondo-Wood G. Review of symptom experiences in children and adolescents with cancer. Cancer Nurs. 2012;35(2):E31-E49. https://doi.org/10.1097/NCC. 0b013e3182207a2a.

3. World Health Organization. Health topics: rehabilitation.. http://www.who.int/topics/rehabilitation/en/. Accessed 12 Dec 2015.

4. UNICEF. Fact sheet: a summary of the rights under the convention of the rights of the child.. http://www.unicef. org/crc/files/Rights_overview.pdf. Accessed 12 Dec 2015.

5. UNICEF.Convention on therights of the child.. http://www. unicef.org/crc/. Accessed 12 Dec 2015.

6. Sperl W, Nemeth C, Fueloep G, Koller I, Vavrik K, Bernert G, et al. Rehabilitation für Kinder und Jugendliche in Österreich. Stand der Dinge und ein Blick über die Grenzen. Monatsschr Kinderheilkd. 2011;159:618-26.

7. Duffner U, Sauter S, Bergstrasser E, Brandis M, Niemeyer C. Oncologic after-care: a patient-oriented concept. Basic diagnostic plan for pediatric oncology patients. Klin Padiatr. 1995;207:193-203. in German.

8. Sperl W. Rehabilitation von Kindern und Jugendlichen in Österreich, ein Blick zurück und nach vorne. Paediatr Paedolog Austria. 2017; https://doi.org/10.1007/s00608017-0522-9.

9. Holzner B, Giesinger JM, Pinggera J, et al. The Computer based Health Evaluation Software (CHES): a software for electronic patient-reported outcome monitoring. BMC Med Inform Decis Mak. 2012;12:126.

10. Varni JW, Seid M, Kurtin PS. PedsQL 4.0: reliability and validity of the pediatric quality of life inventory version 4.0 generic core scales in healthy and patient populations. Med Care. 2001;39(8):800-12. https://doi.org/10.1097/ 00005650-200108000-00006. PMID: 11468499.

11. Varni JW, Burwinkle TM, Katz ER, Meeske K, Dickinson P. The PedsQL in pediatric cancer: reliability and validity of the pediatric quality of life inventory generic core scales, multidimensional fatigue scale, and cancer module. Cancer. 2002;94(7):2090-106. https://doi.org/10.1002/cncr. 10428. PMID: 11932914.

12. Ellis PD. The essential guide to effect sizes: statistical power, meta-analysis, and the interpretation of research results. Cambridge, New York: Cambridge University Press; 2010.

13. Tanner L, Keppner K, Lesmeister D, Lyons K, Rock K, Sparrow J. Cancer rehabilitation in the pediatric and adolescent/young adult population. Semin Oncol Nurs. 2020;36(1):150984. https://doi.org/10.1016/j.soncn.2019. 150984. PMID:31983485.

14. Krauth KA. Family-oriented rehabilitation (FOR) and rehabilitation of adolescents and young adults (AYA) in pediatric oncology. Oncol Res Treat. 2017;40(12):752-8. https://doi. org/10.1159/000484609. PMID:29151110.

15. Tsao E, Bjornson K, Christensen A, Apkon S. Functional outcomes and unplanned transfers of pediatric patients with central neurological impairments receiving inpatient rehabilitation care with cancer and noncancer diagnoses. PM R. 2016;8(6):529-35. https://doi.org/10.1016/j.pmrj. 2015.10.007. PMID:26514788.

16. Eiser C,Varni JW. Health-related quality of life and symptom reporting: similarities and differences between children and their parents. Eur J Pediatr. 2013;172(10):1299-304.

17. Ravens-Sieberer U, Karow A, Barthel D, Klasen F. How to assess quality of life in child and adolescent psychiatry. Dialogues Clin Neurosci. 2014;16:147-58. 
Publisher's Note Springer Nature remains neutral with regard to jurisdictional claims in published maps and institutional affiliations.

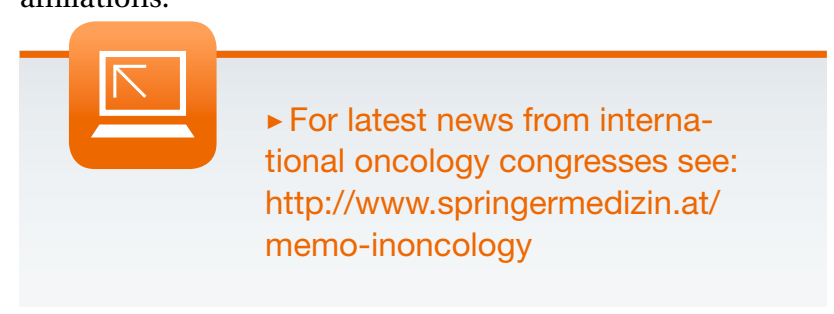

